\title{
Análisis del conocimiento, uso y actitud de las TIC por parte de Maestros de Educación Especial
}

\author{
Lorena Galiano-Barrocal, Pilar Sanz-Cervera y Raúl Tárraga-Mínguez. Universidad de Valencia \\ Recepción: 23 de octubre de 2015 | Revisión: 23 de octubre de 2015 | Aceptación/Publicación: 25 de octubre de 2015 \\ Correspondencia: logaba@alumni.uv.es | pilar.sanz-cervera@uv.es | raul.tarraga@uv.es \\ Citar: Galiano-Barrocal, L., Sanz-Cervera, P. y Tárraga-Mínguez, R. (2015). Análisis del conocimiento, uso y actitud de las TIC por \\ parte de Maestros de Educación Especial. ReiDoCrea, 4, 359-369. [http://hdl.handle.net/10481/38588]
}

\begin{abstract}
Resumen: Antecedentes: Las Tecnologías de la Información y la Comunicación (TIC) son actualmente una herramienta esencial para trabajar con los alumnos con necesidades específicas de apoyo educativo. Las TIC se convierten en una herramienta especialmente valiosa cuando son los propios docentes quienes adaptan el contenido a las características de cada alumno. Objetivos: el presente estudio realiza una revisión de los programas de autor más utilizados y analiza el uso de las TIC que realizan los maestros de educación especial. Método: 16 maestros de pedagogía terapéutica de diferentes centros cumplimentaron un cuestionario elaborado por los autores del estudio. Resultados: los maestros poseen un conocimiento muy bajo sobre los programas de autor, de manera que realizan un uso superficial de los mismos, sin llevar a cabo una creación personalizada y adaptada a las necesidades de los alumnos. Además, se observa que la formación pedagógica de los maestros es insuficiente y que un aumento en la formación tecnológica no conlleva un incremento en el uso de las TIC. Conclusiones: el presente trabajo evidencia que debe haber una mejor formación del profesorado de educación especial en el uso de las TIC.
\end{abstract}

Palabras clave: Educación Especial | Tecnologías de la Información

Analysis of knowledge, use and attitude of ICT tools by Special Education Teachers

\begin{abstract}
Introduction: Information and Communication Technologies (ICT) are nowadays an essential tool for working with students with special education needs. ICT are especially effective when teacher adapt their contents to the characteristics of students. Method: sixteen special education teachers completed a questionnaire prepared by the study's authors. Results: teachers have a very low knowledge of educational software, doing a surface use of them, without carrying out a creation in order to personalize and adapt them to the pupils' needs. In addition, it is noted that the pedagogical training of teachers is inadequate and an increase in technological training does not involve an increase in the use of ICT. Conclusions: this work evidences that it is necessary to get a deeper training of future special education teachers in the use of ICT.
\end{abstract}

Key words: Special Needs Education | Information Technology

\section{Introducción}

En la actual era digital, las Tecnologías de la Información y Comunicación (TIC) juegan un papel cada vez más relevante en todos los aspectos de la vida. Todos los avances ligados a las TIC se extienden a una velocidad vertiginosa provocando grandes cambios en diferentes sectores sociales, y entre ellos, el ámbito educativo. Sin embargo para los maestros de Pedagogía Terapéutica (PT) las TIC juegan un papel todavía más importante que para el resto del profesorado debido: por una parte, a la reducida ratio de alumnado con que intervienen (lo que posibilita un mayor uso de las TIC), y por otra parte, a las características especiales de estos alumnos, ya que en muchos casos las TIC pueden ayudar a que estos estudiantes a accedan a los objetivos y contenidos a través de los canales visual, auditivo e incluso táctil de un modo más sencillo que a través del tradicional material impreso. Como afirma Gómez (2012), para este colectivo es muy importante el uso de las TIC en las aulas, ya que estos instrumentos son capaces de adaptarse a las necesidades, siendo respetuosos con la diversidad, demanda y características particulares de cada persona y facilitando el acceso a la información y a la comunicación a aquellos que más dificultades tienen. En esta línea, Ramírez, Rodríguez y Clemente (2007, p.351) exponen que "las TIC aumentan las capacidades comunicativas, expresivas, analíticas y lógicas del entorno de enseñanza/aprendizaje, además de apoyar experiencias y aprendizajes que serían difíciles por otras vías". 
No obstante, a pesar de los avances tecnológicos, existen déficits en el conocimiento y utilización de los mismos por parte del profesorado. Como señalan Hernández y Quintero (2009), las TIC con sus virtudes pero también con sus limitaciones, se han asentado en el ámbito educativo pero no han venido acompañadas de una nueva concepción o cambio de los modelos de enseñanza y ni siquiera han repercutido en la actividad de aprendizaje de los alumnos.

Ante esta situación, los docentes tienen la necesidad de reinventarse. Su preocupación actual se centra en cómo incorporar las TIC como parte de un proceso de innovación pedagógica, lo cual requiere ser conscientes de la necesidad de un cambio metodológico que afecte al proceso de enseñanza/aprendizaje, empezando por la asimilación y creación de un nuevo rol docente y la implicación de este en una formación permanente de calidad, que permita la incorporación de las TIC de forma significativa.

Nuevo rol docente. Las tecnologías están propiciando que el rol del maestro sea cada vez más complejo, lo cual requiere nuevas funciones $y$, por tanto, nuevas pedagogías y nuevos planteamientos en su formación tanto inicial como permanente (Domínguez, 2012). Al acceder a un aula multimedia el alumno cambia su forma de aprender y el profesor debe modificar su rol, procurando que se lleve a cabo un proceso de enseñanza/aprendizaje bidireccional, marcado por la participación activa del alumno. El papel del docente, en este caso, pasa de ser de un transmisor de información a un facilitador y proveedor de recursos en un entorno colaborativo de aprendizaje, además de un organizador dinámico del currículum. En este contexto, un profesor competente debe ser capaz de adaptar el currículum en función de las necesidades educativas, capacidades individuales y ritmo de aprendizaje de sus alumnos (Gómez et al., 2002); sin olvidar que es más importante prestar atención a los aspectos pedagógicos que a los meramente técnicos, ya que sólo con equipamientos e infraestructura no es posible generar aprendizajes significativos. Para ello, como señala Cabero (2004), el docente no sólo debe ser un consumidor de medios, sino un diseñador y productor de recursos, adaptados a las necesidades de los estudiantes. En esta línea, surgen las herramientas de autor, una pedagogía centrada en los intereses del niño, donde de forma personalizada se les atiende a través de las TIC.

La formación del profesorado. Hernández y Quintero (2009) consideran que el factor clave para la integración de los medios tecnológicos en la enseñanza es la formación del profesorado pero es necesario que dicha formación vaya más allá de la alfabetización tecnológica y contemple el desarrollo de competencias en el profesorado de cara a optimizar el uso didáctico de los medios. Como revela una investigación sobre buenas prácticas en los centros TIC, la mayoría de la formación de los docentes está más extendida en ofimática que en metodología (Rodríguez y Pozuelos, 2009). De este modo, como señala Sáez (2011), sólo uno de cada cinco docentes dice tener seguridad técnica o didáctica para hacer uso de webquests, blogs, programas de edición de imagen o manejo del sistema Linux. Ante esta situación, es evidente que existen importantes deficiencias en la formación didáctica del profesorado, la cual debe estar estrechamente relacionada con las necesidades y problemas reales de cada aula. Por tanto, después de una alfabetización tecnológica inicial, los docentes actuales requieren una formación más pedagógica que les permita tomar decisiones en torno a cómo se pueden aplicar los distintos recursos tecnológicos, cuáles pueden ser sus posibles funciones en situaciones educativas diversas y aplicar estrategias de uso e incorporación curricular de las TIC. Especialmente se necesita formación en aquellas tareas que se refieren al empleo de las tecnologías de ayuda y/o software educativo, así como las relacionadas con el diseño de situaciones de aprendizaje y actividades utilizando las TIC. 
Los programas de autor. La utilización de los programas de autor abiertos presenta una ventaja fundamental frente al uso de materiales cerrados, ya que los primeros permiten personalizar, modificar y adecuar el contenido de los materiales a las características concretas de cada estudiante o grupo de estudiantes, mientras que los segundos son recursos previamente diseñados por el creador para que el usuario lo utilice directamente sin que pueda modificar su contenido. Las herramientas de autor son por tanto programas de diseño que facilitan la creación de actividades educativas, utilizando esquemas prediseñados que proporciona el propio software y que el profesorado puede personalizar y adaptar a las características y necesidades de sus estudiantes, teniendo en cuenta las capacidades y el ritmo de aprendizaje de estos. Además, una gran cantidad de ellos se distribuye como software educativo libre, bajo licencias gratuitas que permiten su libre uso y distribución. Como expone Gómez et al. (2002), el uso de herramientas de autor para generar materiales multimedia supone en sí, una forma inequívoca de integración curricular de las TIC, que debe ser alentada e impulsada por nuestras autoridades educativas de forma clara y decidida. No hace falta tener excesivos conocimientos informáticos para la elaboración de potentes programas, aunque es preciso cierto grado de formación previa y disponibilidad de tiempo.

El presente trabajo persigue dos objetivos: 1) Realizar una revisión detallada, aunque no exhaustiva, de los diferentes programas de autor de que disponen los maestros de Pedagogía Terapéutica para trabajar los contenidos curriculares. 2) Analizar el uso de las TIC que realiza el profesorado de Pedagogía Terapéutica de la localidad alicantina de Ibi. Mediante este objetivo se pretende: a) Conocer los recursos tecnológicos de que disponen los maestros de PT; b) Conocer su formación y competencias en TIC; c) Investigar qué recursos TIC son usados por el profesorado; y, d) Percibir la actitud de estos hacia las TIC.

\section{Material y Métodos}

\section{Participantes}

Participaron 16 maestros de Pedagogía Terapéutica, quienes trabajaban en diferentes centros educativos de la localidad de lbi (Alicante) durante el curso escolar 2012/2013. De los 16 maestros, el $93.75 \%$ eran maestros de Pedagogía Terapéutica y sólo el $26.6 \%$ de estos tenían otras titulaciones como la Licenciatura de Psicopedagogía o la titulación de Maestro de otra especialidad. En la Tabla 1 se incluye la media de edad y años de experiencia de los docentes, considerando si su situación laboral era de definitiva o interinos.

\begin{tabular}{ccc}
\hline \multicolumn{3}{c}{ Tabla 1. Descripción demográfica de la muestra } \\
\hline Situación laboral & $\begin{array}{c}\text { Edad } \\
\text { Media (DT) }\end{array}$ & $\begin{array}{c}\text { Años de experiencia docente } \\
\text { Media (DT) }\end{array}$ \\
\hline Definitiva & $38.6(8.1)$ & $12(6.34)$ \\
Interina & $31(1.4)$ & $2.95(2.9)$ \\
\hline Total & $37.6(8)$ & $10.87(6.7)$ \\
\hline
\end{tabular}

Para realizar el estudio se contó con la colaboración de 8 centros educativos: 5 centros públicos (CEIP), 2 centros de régimen concertado y 1 centro de educación especial (CEE). El alumnado que asistía a las aulas de PT de estos 8 centros 
ascendía a 156 alumnos, con una media de 9.8 alumnos por maestro; aunque la distribución era considerablemente desigual entre los centros, a excepción del Centro de Educación Especial donde cada PT contaba entre 5 y 9 alumnos con características similares. En cuanto al tipo de discapacidad de los alumnos, predominan las discapacidades cognitivas con un $81.25 \%$, seguido de las discapacidades motrices en un $56.25 \%$, otro $56.25 \%$ lo engloba los Trastornos del Neurodesarrollo (como TEA y/o TDAH) y, finalmente, las discapacidades sensoriales en un $25 \%$.

\section{Instrumento de evaluación}

Para realizar el estudio se ha diseñado un cuestionario compuesto por 73 ítems, divididos en 7 bloques, considerando los aspectos más importantes que incluyen las últimas investigaciones al respecto (Ramírez et al., 2007; Roig, Ferrández, Rodríguez y Crespo, 2012; Sáez, 2011). Los 7 bloques son: 1) El perfil del PT y su contexto laboral; 2) Equipamiento informático y disponibilidad de recursos; 3) Uso de las TIC en el contexto educativo; 4) Metodología de implantación de las TIC; 5) Herramientas de autor para el diseño de actividades educativas; 6) Formación y competencias en TIC del PT; 7) Nuevos retos ante las TIC. En cada uno de los bloques se incluyen entre 5 y 18 ítems.

\section{Procedimiento}

En primer lugar, se realizó una búsqueda bibliográfica para analizar los programas de autor de que disponen los docentes. En segundo lugar, para analizar el uso de las TIC que realizan los docentes, se elaboró el cuestionario descrito anteriormente. La recogida de los datos se realizó de forma personal, aplicando el cuestionario en formato entrevista. Una vez recogidos los datos, se realizaron análisis descriptivos y de distribución de frecuencias mediante el paquete estadístico SPSS-19.

\section{Resultados}

Objetivo 1: Revisión de los programas de autor. Después de diferentes búsquedas bibliográficas en buscadores y sitios web oficiales de educación de las diferentes comunidades autónomas, se localizaron siete programas de autor, como son: Ardora, Constructor, JClic, Cuadernia, Edilim, Malted y Rayuela. A continuación se incluye una breve descripción de los mismos, así como una síntesis de las principales características con los enlaces a los respectivos sitios web, bases de datos y tutoriales (Tabla 2).

Ardora: Desarrollado por José Manuel Bouzán, se puede descargar y utilizar gratuitamente. Ofrece la posibilidad de realizar 45 tipos de actividades diferentes agrupadas en diversos bloques: actividades con gráficos, juegos de palabras, actividades con sonido, de clasificar, relacionar, completar, ordenar, seleccionar, tests, esquemas, actividades con gráficos y estadísticos, unidades de medida, cálculo y geometría.

Constructor: Elaborado por la Consejería de Educación y Cultura de la Junta de Extremadura. Se distribuye bajo licencia Creative Commons-Reconocimiento no comercial-Compartir igual. Este programa permite la elaboración de actividades a partir de 53 plantillas diferentes, agrupadas en tres bloques: plantillas de evaluación, plantillas lúdicas y plantillas avanzadas.

JClic: Creado por la Xarxa Telemática Educativa de Cataluña, se puede descargar de manera gratuita. Su principal ventaja es que presenta una amplia base de datos de actividades de diferentes materias y niveles. Contempla actividades de: asociación simple, asociación compleja, pantalla de información, actividad de exploración, actividad de identificación, juego 
de memoria, puzle doble, puzle de intercambio, puzle de agujero, crucigrama, sopa de letras, actividades de respuesta escrita y actividades con texto. Adicionalmente, JClic posee el módulo "arith" que puede aplicarse a diferentes tipos de actividades.

Cuadernia: Desarrollado por la Consejería de Educación, Cultura y Deportes de CastillaLa Mancha, bajo licencia Creative Commons. Presenta una estructura que le diferencia de otros programas de autor, y es que permite en una misma pantalla introducir diferentes tipos de elementos y actividades, simulando un libro "tradicional", pero con la ventaja de que permite la interacción.

Edilim: Creado por Fran Macías. Según se indica en la web es de uso y distribución libre, siempre que se respete su gratuidad y autoría. Proporciona 51 tipos de actividades, que el propio programa clasifica en 5 categorías de actividades: De información, con palabras, con imágenes, con números y juegos.

Malted: Realizado por profesionales de la educación de diferentes países de la Unión Europea. En España recibe soporte por parte del Instituto de Tecnología Educativa (ITE) del Ministerio de Educación, que lo distribuye bajo licencia CreativeCommonsReconocimiento-No comercial-Compartir bajo la misma licencia. Proporciona 13 tipos de plantillas para el diseño de actividades.

Rayuela: Desarrollado por el Instituto Cervantes. Según indica su página web esta herramienta está registrada y es propiedad del Instituto Cervantes, el cual la distribuye gratuitamente para su libre utilización con fines docentes. Proporciona la posibilidad de diseñar 12 tipos de actividades: juego del ahorcado, crucigrama, cuadro de mandos, hilo conductor, juego de lógica, opción múltiple, relacionar listas, rellenar huecos, rompecabezas, salto del caballo, rompecabezas y verdadero-falso.

Tabla 2. Principales características de las herramientas de autor revisadas

\begin{tabular}{|c|c|c|c|c|c|c|}
\hline Programa & $\begin{array}{l}\text { Creador / } \\
\text { Institución }\end{array}$ & Sitio web & Versiones & $\begin{array}{c}\text { Tipo de } \\
\text { instalación }\end{array}$ & Base de datos & Tutoriales \\
\hline Ardora & José Manuel Bouzán & http://webardora.net/ & $\begin{array}{c}\text { Ubuntu } \\
\text { Windows }\end{array}$ & Versión portable & $\begin{array}{c}\text { http://webardora.net/exempl } \\
\text { os_cas.htm }\end{array}$ & $\begin{array}{c}\text { http://webardora.net/axuda_cas } \\
\text {.htm }\end{array}$ \\
\hline Constructor & $\begin{array}{l}\text { Consejería de } \\
\text { Educación y Cultura de } \\
\text { la Junta de Extremadura }\end{array}$ & $\begin{array}{c}\text { http://constructor.educarex.e } \\
\mathrm{s} /\end{array}$ & $\begin{array}{l}\text { Ubuntu } \\
\text { Windows }\end{array}$ & Instalable & $\begin{array}{l}\text { http://constructor.educarex. } \\
\text { es/index.php?option=com_r } \\
\text { emository\&ltemid=29 }\end{array}$ & $\begin{array}{l}\text { http://constructor.educarex.es/in } \\
\text { dex.php?option=com_content\&t } \\
\text { ask=view\&id=33\&ltemid=68 }\end{array}$ \\
\hline Cuadernia & $\begin{array}{l}\text { Consejería de } \\
\text { Educación, Cultura y } \\
\text { Deportes de la Junta de } \\
\text { Comunidades de } \\
\text { Castilla-La Mancha } \\
\end{array}$ & $\begin{array}{l}\text { http://www.educa.jccm.es/ed } \\
\text { uca- } \\
\text { jccm/cm/recursos/tkContent } \\
\text { ?idContent=70656\&textOnly } \\
\text { =false\&locale=es_ES }\end{array}$ & $\begin{array}{l}\text { Linux } \\
\text { Windws }\end{array}$ & $\begin{array}{l}\text { En línea, instalable, } \\
\text { portable }\end{array}$ & $\begin{array}{l}\text { http://www.educa.jccm.es/e } \\
\text { duca- } \\
\text { jccm/cm/recursos/tkContent } \\
\text { ?idContent=70674\&locale= } \\
\text { es_ES\&textOnly=false }\end{array}$ & $\begin{array}{c}\text { http://www.educa.jccm.es/educ } \\
\text { a- } \\
\text { jccm/cm/recursos/tkContent?id } \\
\text { Content=70675\&locale=es_ES } \\
\text { \&textOnly=false }\end{array}$ \\
\hline JClic & $\begin{array}{c}\text { Xarxa Telemàtica } \\
\text { Educativa de Catalunya } \\
(\text { Xtec })\end{array}$ & $\begin{array}{c}\text { http://clic.xtec.cat/es/index.ht } \\
\text { m }\end{array}$ & $\begin{array}{l}\text { Linux, Mac, Solaris, } \\
\text { Windows }\end{array}$ & Instalable & $\begin{array}{l}\text { http://clic.xtec.cat/db/listact_ } \\
\text { es.jsp }\end{array}$ & $\begin{array}{c}\text { http://clic.xtec.cat/es/jclic/curs.ht } \\
\mathrm{m}\end{array}$ \\
\hline Malted & $\begin{array}{l}\text { Instituto de Tecnología } \\
\text { Educativa (ITE) del } \\
\text { Ministerio de Educación, } \\
\text { Cultura y Deporte }\end{array}$ & $\begin{array}{l}\text { http://recursostic.educacion. } \\
\text { es/apls/informacion_didactic } \\
\text { a/617 }\end{array}$ & Linux Windows & Instalable & $\begin{array}{l}\text { http://recursostic.educacion. } \\
\text { es/malted/web/recursos/ind } \\
\text { ex.htm }\end{array}$ & $\begin{array}{c}\text { http://recursostic.educacion.es/ } \\
\text { malted/web/Curso_Malted_ava } \\
\text { nzado/Entrada_al_curso.htm }\end{array}$ \\
\hline Rayuela & Instituto Cervantes & $\begin{array}{l}\text { http://cvc.cervantes.es/aula/ } \\
\text { pasatiempos/ }\end{array}$ & Windows & Instalable & $\begin{array}{l}\text { http://cvc.cervantes.es/aula/ } \\
\text { pasatiempos/pasatiempos2/ }\end{array}$ & $\begin{array}{c}\text { http://www.cervantes.es/lengua } \\
\text { _y_ensenanza/tecnologia_espa } \\
\text { nol/rayuela.htm }\end{array}$ \\
\hline
\end{tabular}


Objetivo 2: Analizar el uso de las TIC que realiza el profesorado de PT. Dentro de este objetivo general, se plantearon diferentes análisis, que se detallan a continuación:

a) Conocer la formación y competencias en TIC de los maestros de PT: El profesorado es consciente de su escasa formación en TIC. De hecho, nadie se considera experto usando las tecnologías. Como se muestra en la Figura 1, el $62.5 \%$ considera poseer una formación básica de usuario, el $25 \%$ considera que su formación es baja y tan solo el $12.5 \%$ considera poseer una formación avanzada. La mitad de los maestros consideran que, a pesar de su escasa formación, existe una buena oferta de formación para que los maestros de PT puedan aprender a utilizar las TIC con fines didácticos. Además, la totalidad de los docentes considera que es muy importante fomentar el uso de las TIC y el $93.75 \%$ presentan una actitud positiva hacia el trabajo colaborativo entre el personal docente universitario y los maestros de PT, con la finalidad de innovar en el uso de las TIC y poder realizar una práctica pedagógica más eficaz.

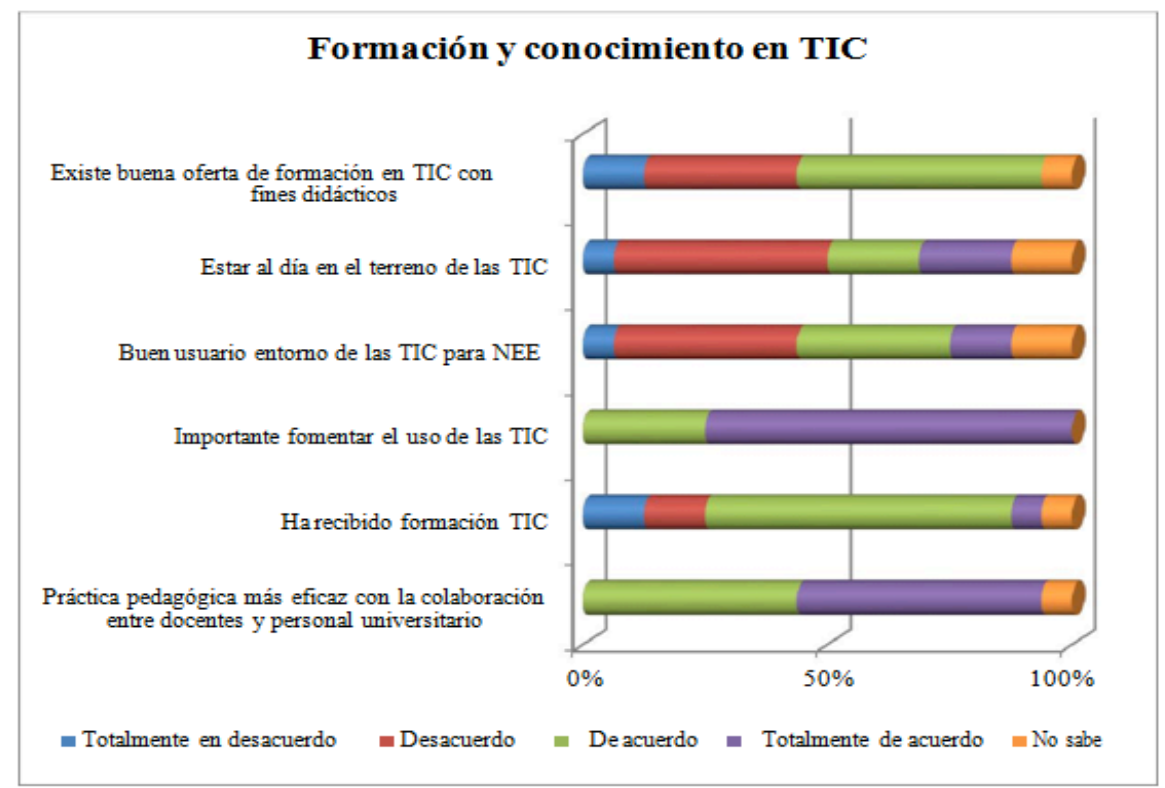

Figura 1. Formación y conocimiento de las TIC por parte de los maestros de PT

Del total de los 16 docentes entrevistados, 7 afirman utilizar programas de autor, 5 los conocen pero no los usan en el aula y los 4 restantes no poseen conocimiento de los mismos. De los siete programas revisados en este trabajo, el $68.75 \%$ de los PT afirman usar en su aula el programa JClic. De ellos, el $18.75 \%$ lo hacen diariamente, el $37.5 \%$ más de dos veces al mes y el $12.5 \%$ solo lo utilizan una vez al año. Con menor frecuencia algunos maestros utilizan otros de los programas revisados, como el Edilim y el Rayuela que son utilizados por un maestro en cada caso y el Cuadernia que es utilizado por dos de los maestros. El resto de programas revisados, como son el Ardor, Constructor y Malted, no se usan en las aulas (Véase Figura 2). 


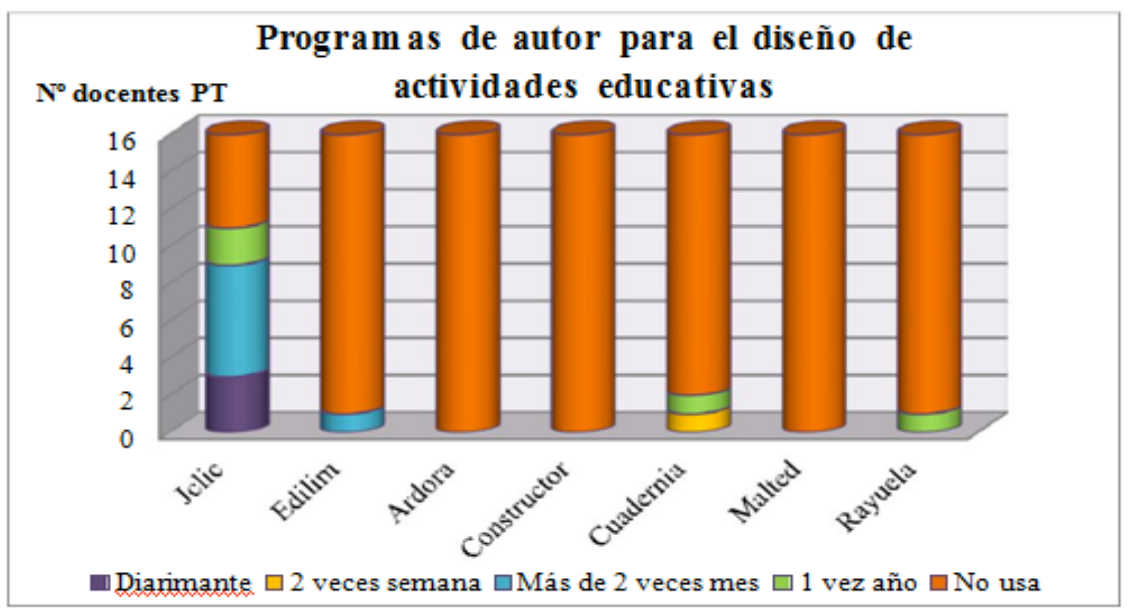

Figura 2. Frecuencia de uso de los programas de autor

b) Recursos TIC que utiliza el profesorado: En la Figura 3 se observa que la herramienta que más usan los docentes diariamente es el procesador de texto (56.25\%), seguido del correo electrónico de uso personal y aplicaciones educativas para los alumnos en un $43.75 \%$. Lo que menos realizan a diario es la elaboración de materiales con las TIC $(6.25 \%)$. Los docentes admiten con un $87.5 \%$ que no usan las TIC para el diseño de webs, blogs, webquests, etc. No obstante, todos admiten, aunque con distinta frecuencia de uso, que leen información de la red y navegan por internet prácticamente a diario. Además, el manejo de programas de presentación de diapositivas tipo PowerPoint, imágenes o vídeos se utilizan en el $50 \%$ menos de una vez al mes.

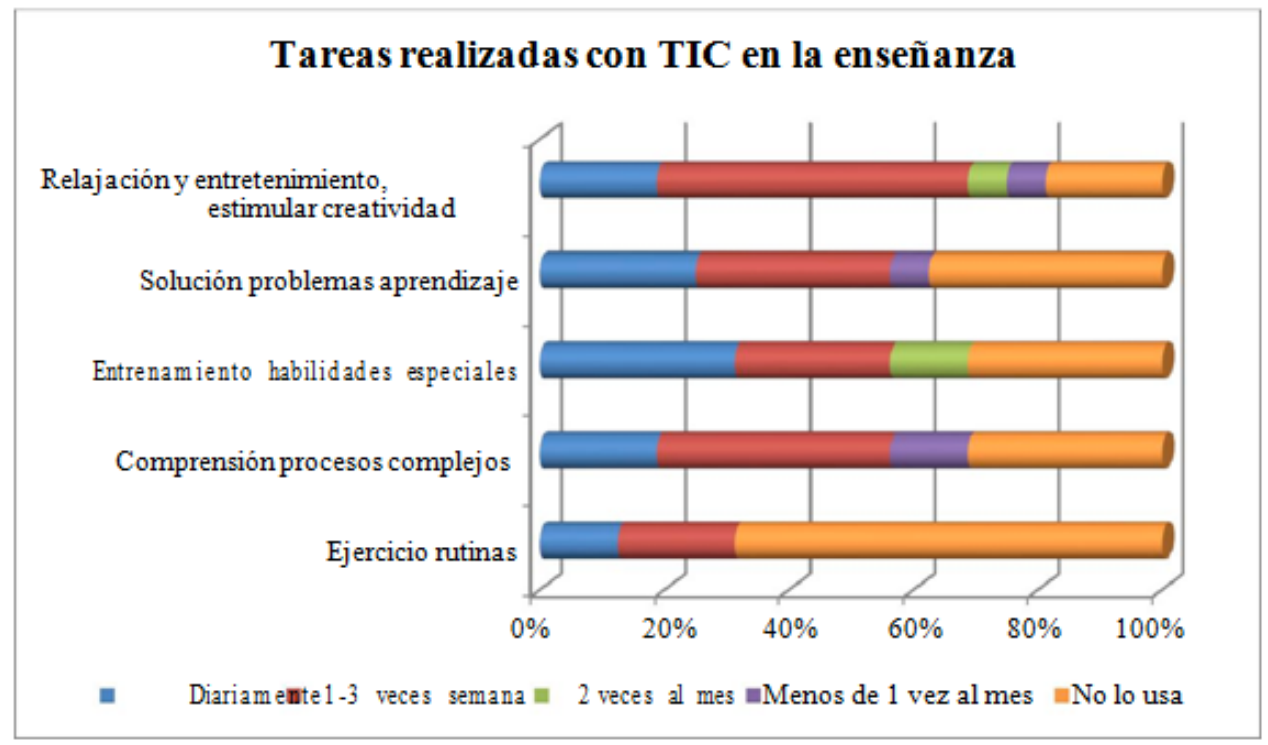

Figura 3. El uso de las TIC en la preparación de la docencia

c) Actitud de los docentes hacia las TIC: Según los resultados obtenidos, la actitud del profesorado hacia las TIC es muy positiva, ya que la totalidad de los maestros están muy de acuerdo en que las TIC pueden ayudar a los alumnos con NEAE en su aprendizaje; además de considerar que es muy importante la formación continua del profesorado. Un $93.75 \%$ también consideran que deberían ubicarse los recursos TIC en aulas especializadas y que dichos recursos en la escuela son una herramienta fundamental para la enseñanza. A pesar de estos porcentajes tan 
elevados, tan solo un $56.25 \%$ manifiesta que programa e incluye objetivos, contenidos y actividades en relación a las tecnologías en la práctica educativa y un $25 \%$ no están de acuerdo con la programación de este tipo de actividades (Véase Figura 4).

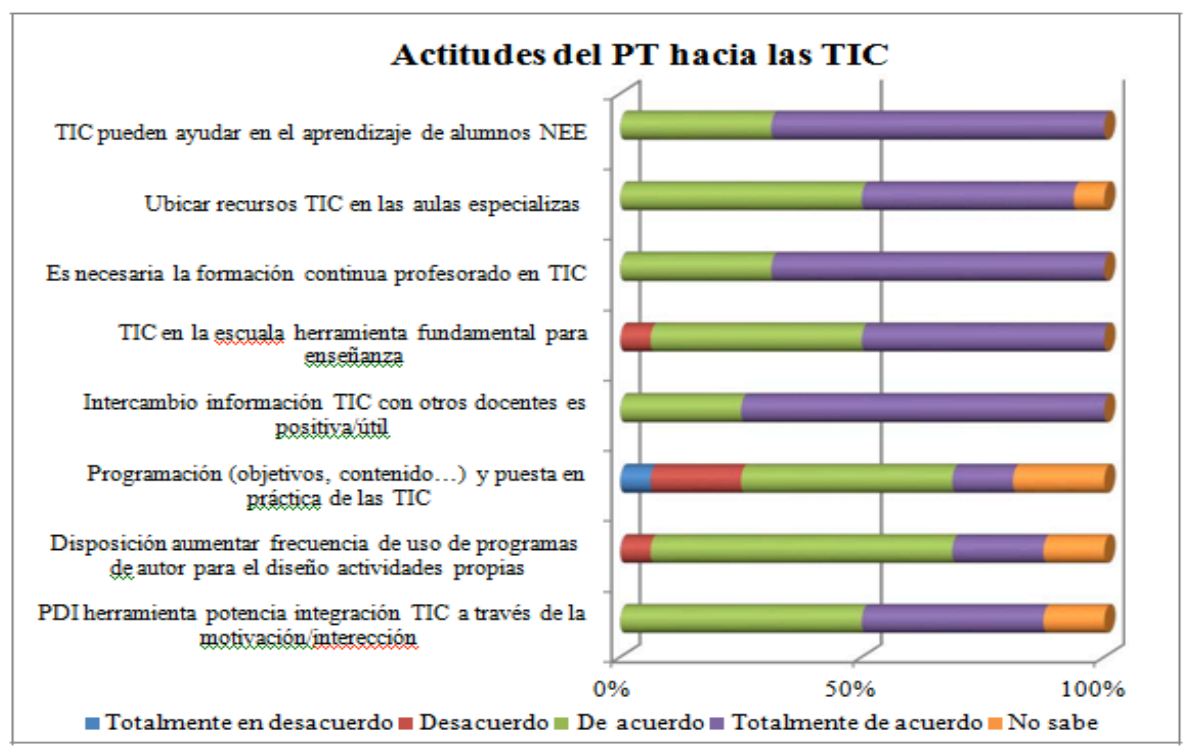

Figura 4. Actitudes del PT hacia las TIC

Como se observa en la Figura 5, uno de los principales motivos por los cuales los docentes no se animan a hacer uso de las TIC es por desconocimiento (93.75\%). Un $62.5 \%$ consideran que el hecho de usar las TIC en las actividades escolares supone emplear más tiempo en la preparación de las clases y en la mayoría de casos exponen que no disponen de dicho tiempo. En cuanto a la dotación económica para conseguir nuevo equipamiento, el $68.75 \%$ considera que es insuficiente, mientras que un $25 \%$ considera que sí que hay dinero para este tipo de adquisiciones.

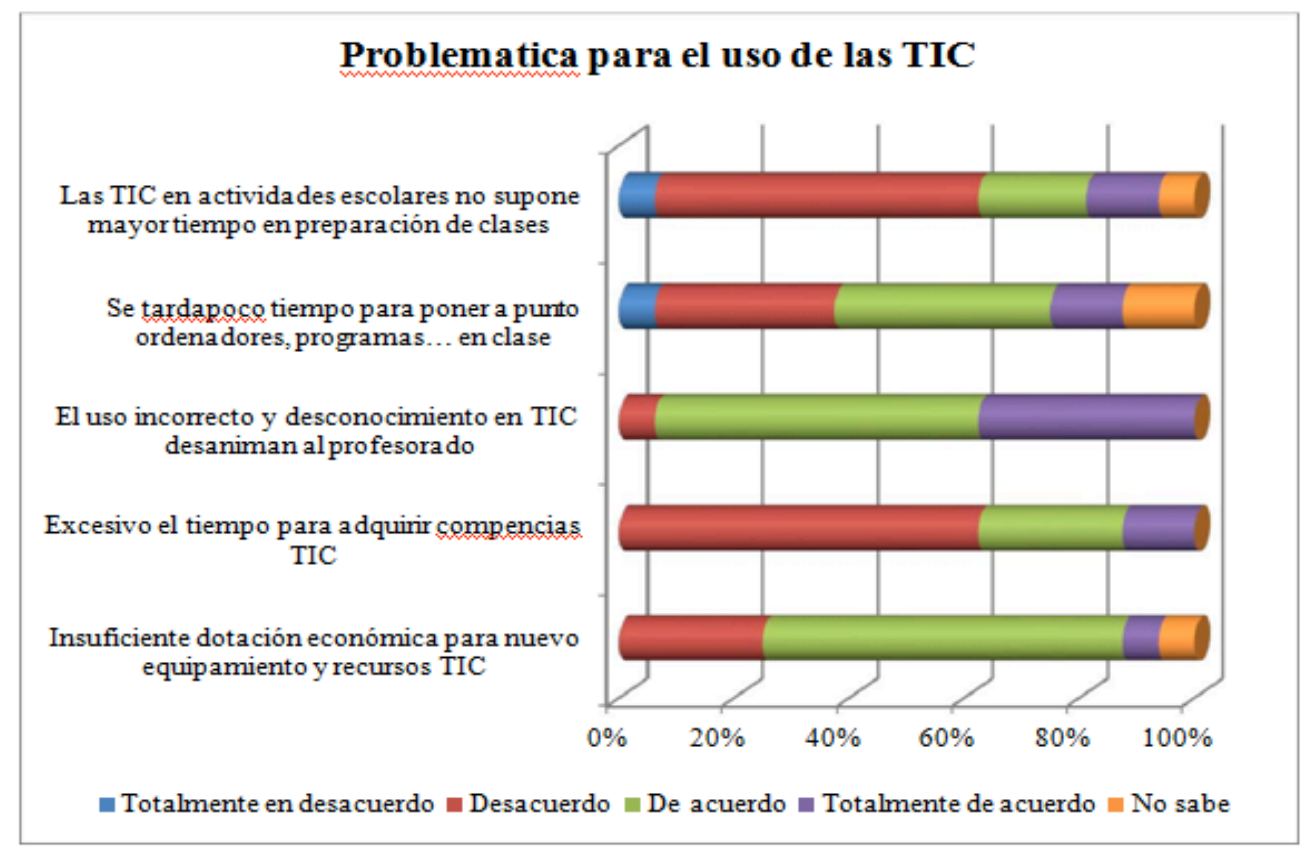

Figura 5. Problemas en el uso de las TIC 


\section{Discusión}

Tras analizar los resultados obtenidos, cabe destacar que el $100 \%$ de los maestros de PT están de acuerdo con que el uso de las TIC puede ayudar en el aprendizaje del alumnado con NEAE. Además, valoran que la formación continua del profesorado es necesaria y que el intercambio de información TIC con otros docentes es útil y positivo para mejorar sus conocimientos en este ámbito.

A pesar de esta positiva valoración, el profesorado es consciente de su falta de formación en TIC. Incluso en los casos de haber recibido dicha formación, los docentes afirman que esta no ha sido adecuada y/o funcional. No obstante, están dispuestos a emplear su tiempo en formación para llegar a ser competentes digitales, ya que afirman estar interesados en ofrecer la mejor oferta educativa a sus alumnos en cuanto a tareas de ayuda y software educativo se refiere. La formación que más les interesa recibir versa sobre todo en aprender a cómo utilizar las pizarras digitales y las tablets, aunque también desean aprender programas y herramientas que faciliten el proceso de enseñanza/aprendizaje.

Es llamativo que, a pesar de que los docentes son conscientes de la necesidad del uso de las TIC, gran parte de ellos desconoce si existe un Plan de Incorporación de las TIC en sus centros. Otro de los aspectos contradictorios es que todos los docentes muestran su interés en incluir las TIC en su método de trabajo pero sólo el $50 \%$ afirman incluirlas en su programación de aula. En estos casos, no obstante, el uso que se realiza es más reproductivo que creativo, ya que los docentes afirman en un $87.5 \%$ que realizan actividades prediseñadas pero no crean sus propios materiales curriculares. De este resultado se infiere que los docentes poseen un conocimiento muy bajo de los programas de autor. Como reportan los propios maestros, el programa más popular es el JClic, el cual es utilizado en la mayoría de los casos para realizar actividades que han diseñado otros compañeros. El uso que se realiza de este programa, por tanto, es superficial, ya que no se lleva a cabo una creación personalizada y adaptada a las necesidades de los alumnos.

Respecto a la metodología y al tipo de tareas realizadas con las TIC, en un estudio similar realizado con maestros de PT, también se obtuvo que gran parte de los docentes están de acuerdo en la necesidad de elaborar recursos TIC personalizados a cada caso de NEAE (Roig et al., 2012). En el citado estudio, el tipo de actividades en las que se hacía un uso más intensivo de las TIC era en los ejercicios de relajación y entretenimiento, además de los ejercicios de rutinas, sin llegar a hacer un uso más específico de las mismas. En otra publicación similar realizada en un Centro de Educación Infantil (Sevillano y Rodríguez, 2013), se corrobora que las TIC no suelen formar parte de la metodología de trabajo ni de las programaciones de aula, ya que los docentes no saben cómo aprovecharse de sus posibilidades y qué medios en concreto pueden ayudar a mejorar el proceso de enseñanza/aprendizaje. Lamentan que falta tiempo para intercambiar ideas, formación y apoyo institucional. Igualmente, afirman que la inversión en infraestructuras y equipamiento es insuficiente para las exigencias educativas planteadas, por lo que los autores de este estudio concluyen que es necesario ajustar la oferta formativa de los docentes con las demandas educativas.

Como señalan Castaño et al. (2004, p.13), "las TIC se utilizan moderadamente para el apoyo de la metodología más tradicional (presentar información) e incluso para acceder a más información o motivar a los estudiantes y facilitar el autoaprendizaje, pero no parece que se introduzcan para trabajar con otro tipo de metodologías más activas y participativas. Esto hace pensar que se utiliza más como material lúdico 
o de apoyo y, en todo caso, fundamentalmente, si no siempre, como herramienta del propio profesor".

En relación a la formación del profesorado y la incorporación de las TIC en el currículum, Ferrero y Martínez (2011) señalan que los docentes de su investigación afirman recibir poco asesoramiento para constituir grupos de trabajo que ayuden a mejorar sus competencias en TIC y al mismo tiempo los centros educativos no contribuyen en incorporar las TIC en el currículum. En esta misma línea, en el estudio de Fernández, Rodríguez y Vidal (2007) se concluye que el profesorado no conoce en profundidad el potencial educativo de las TIC en el aula y señala que la administración educativa se preocupa fundamentalmente de dotar determinados recursos educativos, pero no tanto de formar didáctica y curricularmente sobre los mismos.

El presente estudio incluye ciertas limitaciones debido a que se trabaja con un tamaño muestral reducido, además de que la investigación realizada es de carácter únicamente cuantitativo, por lo que en futuras investigaciones sería conveniente combinar los cuestionaros con otros instrumentos de recogida de datos de carácter cualitativo. También se podría ampliar el campo de investigación analizando nuevos modelos de formación del profesorado, ya que esta necesita un replanteamiento que tenga en cuenta la nueva realidad social y escolar. Por otro lado, también sería interesante realizar un análisis crítico de los medios y las tecnologías de la información con los que cuenta la sociedad actual y trabajar en el perfeccionamiento de nuevas tecnologías específicamente diseñadas para alumnos con NEAE. Además de compartir experiencias e intercambiar prácticas innovadoras, una vía fundamental para prosperar en la implantación de las TIC es potenciar el desarrollo de Aulas 2.0. Estas aulas tienen como objetivo impulsar las TIC, ofertando al profesorado implicado una formación no solo tecnológica sino también didáctica, por lo que mediante ellas se podría incrementar el conocimiento de las herramientas de autor para el diseño de actividades educativas. Para concluir, cabe destacar que el hecho de instalar ordenadores y poder acceder a Internet en los colegios no sirve de nada si no va a acompañado de formación específica del profesorado. Esta formación debe incluir nuevos lenguajes de la comunicación, a través de la pedagogía de la imagen y el uso racional y crítico de los recursos tecnológicos (Sánchez, 2008). Como hemos visto, la implantación de las TIC en los centros educativos no está en absoluto exenta de problemas, más bien al contrario. De momento podemos afirmar que el uso de las TIC está repleto de buenas intenciones, pero que se materializan con mucha dificultad en prácticas educativas inclusivas. Es necesario insistir en la conveniencia de construir un nuevo modelo educativo inclusivo que utilice las TIC como herramienta indispensable para fomentar el aprendizaje significativo y lograr así la integración del centro escolar en la era tecnológica. Además, es imprescindible dar importancia a la preparación de los docentes para que sean consumidores, diseñadores, creadores y productores de medios. Para ello, se debe crear una fórmula colaborativa que permita perfeccionar la práctica educativa a través del constante intercambio comunicativo entre los distintos docentes. De esta manera se ofrece la oportunidad de reflexionar sobre la práctica diaria e impulsar las innovaciones en los procesos de enseñanza/aprendizaje (Lozano, Alcaraz y Colás, 2010).

\section{Referencias}

Cabero, J. (2004). Formación del profesorado en TIC. El gran caballo de batalla. Comunicación y Pedagogía. Nuevas Tecnologías y Recursos didácticos, 195, 27-31.

Castaño, C., Maiz, I., Beloki, N., Bilbao, J., Quecedo, R. y Mentxaka, I. (2004). La utilización de las TIC en la enseñanza primaria y secundaria obligatoria: Necesidades de formación del profesorado. Edutec, 1-15. 
Domínguez, R. (2012). Las TIC en la formación del profesorado. Revista científica electrónica de Educación y Comunicación en la Sociedad del Conocimiento, 12, 168-179.

Fernández, M.D., Rodríguez, J. y Vidal M.P. (2007). TIC y desarrollo profesional del profesorado. El caso de un centro de primaria. Revista Interuniversitaria de Formación del Profesorado, 21(1), 85-110.

Ferrero, S., y Martínez, M.C. (2011). Formación del profesorado en TIC, en la zona de los montes orientales de Granada. Revista Electrónica de Tecnología Educativa (Edutec-e), 37, 1-15.

Gómez, I. (2012). Discapacidad y tecnología: Un reto para el profesorado del siglo XXI en contextos escolares inclusivos. Revista científica electrónica de Educación y Comunicación en la Sociedad del Conocimiento (Etic@net), 12(2), 187-201.

Gómez, M., Franco, A.M., Martínez J., Pastor, P., Marín, S., Camacho, A.R., y Villalba, J. (2002). Herramientas de autor y aplicaciones informáticas para los alumnos con necesidades educativas especiales asociadas a grave discapacidad. Educar en el 2000, 40-45.

Hernández, A., y Quintero, A. (2009). La integración de las TIC en el currículo: necesidades formativas e interés del profesorado. Revista Electrónica Interuniversitaria de Formación de Profesorado (REIFOP), 12(2), $103-119$.

Lozano, J., Alcaraz, S. y Colás, P. (2010). La enseñanza de emociones y creencias a alumnos con trastornos del espectro autista: Una investigación colaborativa. Revista de currículum y formación del profesorado, 12(1), 367-382.

Ramírez, E., Domínguez, A.B. y Clemente, M. (2007). Cómo valoran y usan las Tecnologías de la Información y la Comunicación (TIC) los profesores de alumnos con Necesidades Educativas Especiales (NEE). Revista de Educación, 342, 349-372.

Rodríguez, F.P. y Pozuelos, F.J. (2009). Aportaciones sobre el desarrollo de la formación del profesorado en los centros TIC. Estudio de casos. Revista de Medios y Educación (Pixel-Bit), 35, 33-43.

Roig, R., Ferrández, S., Rodríguez, C., y Crespo, M. (2012). El uso de las TIC en el aula de Educación Especial: Percepción de los maestros. Respuestas flexibles en contextos educativos diversos. En Navarro, J., Fernández, M.T., Soto F.J. y Tortosa, F. (Coords.). Consejería de Educación, Formación y Empleo de Murcia.

Sáez, J.M. (2011). Opiniones y práctica de los docentes respecto al uso pedagógico de las tecnologías de la información y la comunicación. Revista Electrónica de Investigación y Docencia (REID), 5, 95-113.

Sánchez, A. (2008). Las tecnologías de la Información y la Comunicación en la formación del profesorado. Revista Iberoamericana de Educación, 45(3), 1-13.

Sevillano, M.L., y Rodríguez, R. (2013). Integración de tecnologías de la información y comunicación en educación infantil en Navarra. Revista de Medios y Educación (Píxel-Bit), 42, 75-87. 